\title{
Microsurgical Technique of Simultaneous Pancreas/Kidney Transplantation in the Rat: Clinical Experience and Review of the Literature
}

\author{
$\begin{array}{llll}\text { E. Matevossian } & \text { D. Doll } & \text { I. Sinicina } & \text { H. Kern } \\ & \text { C. Bald } & \text { J. Nährig } & \text { b. Stangla }\end{array}$ \\ S. Thorban ${ }^{a}$ N. Hüser ${ }^{a}$

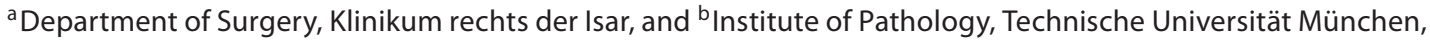 \\ and ${ }^{\mathrm{C}}$ Department of Surgical Research, Ludwig Maximilians University Munich, Munich, Germany
}

\section{Key Words}

Transplantation $\cdot$ Pancreas $\cdot$ Kidney $\cdot$ Allograft $\cdot$ Surgical technique $\cdot$ Rat model

\begin{abstract}
Background: For experimental basic research, standardized transplantation models reflecting technical and immunologic aspects are necessary. This article describes an experimental model of combined pancreas/kidney transplantation (PKTx) in detail. Materials and Methods: Donor rats underwent en bloc pancreatectomy and nephrectomy. Revascularization was performed using the aorta with the superior mesenteric artery and the inferior vena cava with the portal vein. Exocrine drainage of the pancreas took place over a segment of the duodenum which was transplanted side-to-side to the jejunum. The kidney vessels were transplanted end-to-side. The ureter was anastomosed by patch technique. Postoperatively, serum parameters were monitored daily. Biopsies for histopathology were taken on days 5, 8 and 12. Results: All 12 recipients survived the combined PKTx without serious surgical complications. One thrombosis of the portal vein led to organ failure. Blood glucose levels were normal by the $3 \mathrm{rd}$ postoperative day. The transplanted duodenal segment showed slight villous atrophy, and the kidneys were well perfused without vascular complications.
\end{abstract}

The anastomosis between ureter and bladder was leakproof. Conclusions: Excellent graft function and survival rates can be achieved due to simplified operation technique and short operation time. It may thus have high clinical relevance to immunologic issues within the scope of basic research.

Copyright $\odot 2009$ S. Karger AG, Basel

\section{Introduction}

Experimental microsurgical technique using intraoperative microscopy enables abdominal operations and organ transplantations to be performed in rats. Continuing research in microsurgery has considerably expanded experimental surgery and given new impulses to clinical surgery [1-4]. Whole-organ pancreas transplantation in the rat was first described by Lee et al. [3, 4] in 1972. Since that time the basic technique has been modified and extended to be used for a variety of indications and experiments. Simultaneous en bloc pancreaticoduodenal kidney transplantation is being applied with increasing frequency in the treatment of diabetes mellitus type I and selected cases of type II [5-8]. A review of the literature of isolated pancreas transplantation in rat models showed that different techniques or modifications can be applied $[2,4,9,11-13]$. However, pancreas transplantation in the

\section{KARGER}

Fax +4161306 1234 E-Mail karger@karger.ch www.karger.com (c) 2009 S. Karger AG, Basel

$0014-312 X / 09 / 0432-0245 \$ 26.00 / 0$

Accessible online at:

www.karger.com/esr
Norbert Hüser, MD

Department of Surgery, Klinikum rechts der Isar

Technische Universität Munich, Ismaningerstrasse 22

DE-81675 Munich (Germany)

Tel. +49894140 5195, Fax +49894140 4815, E-Mail hueser@chir.med.tu-muenchen.de 
Fig. 1. After removing the pancreas from the intestinal adhesions the duodenum was ligated below the head of the pancreas and removed.

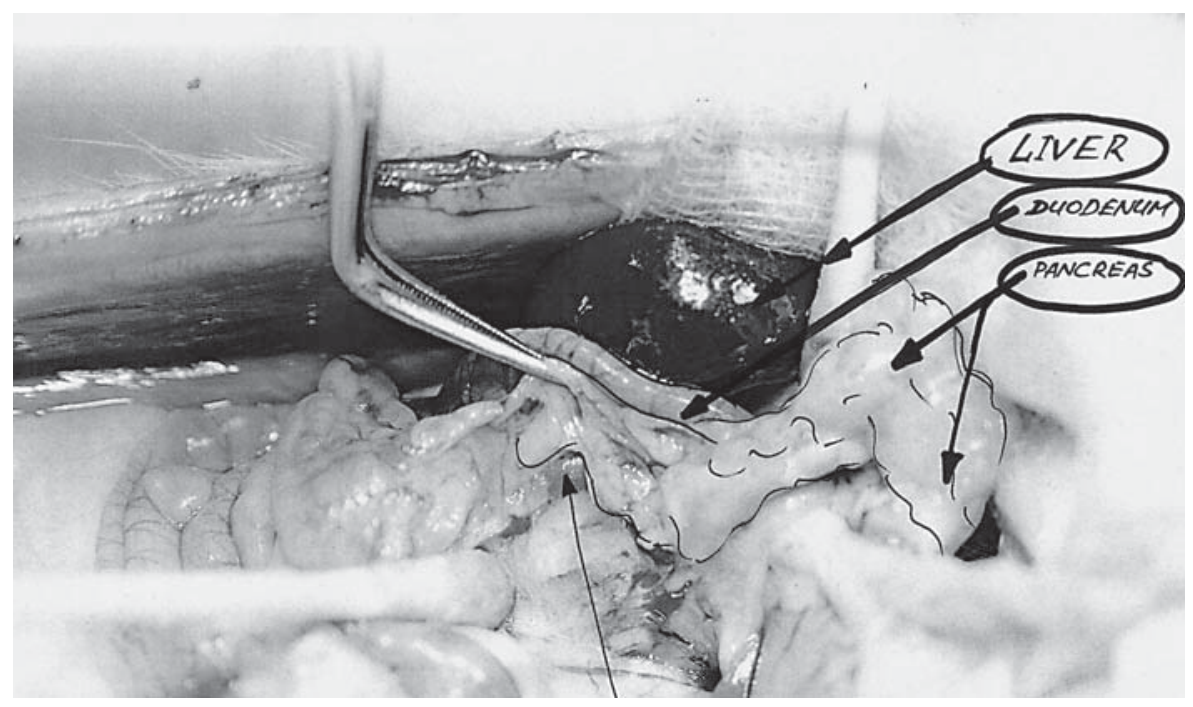

rat remains a complex technical procedure and requires profound knowledge of microsurgical techniques [13]. A surgical technique in extension of this - simultaneous combined pancreas/kidney transplantation (PKTx) - is described here. This novel surgical technique for combined PKTx is carried out in a rat model that closely resembles the anatomic-functional situation of a pancreatic/kidney graft in man. The technique requires a microanastomosis between the portal and right renal veins with a circular cava patch of the donor organ and cava vein of the recipient. Morphological aspects of simultaneously isografted organs are unchanged when compared with separately transplanted organs. This technique provides a useful experimental model for studying several technical, functional and histopathological aspects in combined PKTx in diabetic patients. It is also useful for studying the early phases of ischemic/reperfusion graft injuries. The presented microsurgical technique is reliable and reproducible - and it represents the first reported experimentally combined PKTx. This technique for combined PKTx in the rat may be adopted according to surgeons' experiences and preferences, as well as their research objectives.

\section{Materials and Methods}

\section{Animals}

For donor and recipient operations male Lewis (RT) rats (Charles-River Wiga GmbH, Sulzfeld, Germany) were used to avoid graft rejection. The body weight of the rats was $140 \pm 20 \mathrm{~g}$, whereas the weight of the recipient was $20 \mathrm{~g}$ more than the donor weight, which makes combined PKTx easier.
Preoperative Care and Anesthesia

The rats were kept in Macrolon cages (Ehret GmbH, Emmendingen, Germany) at a room temperature of $19-24^{\circ} \mathrm{C}$ and illuminated from 6 a.m. until 6 p.m. They were fed with water and pellets (Altromin 1324 standard diet, Lage, Germany). Rats were fasted for $4 \mathrm{~h}$ before surgery. Inhalation anesthesia was administered by a semiopen system with isoflurane via nasal mask. The mask had been designed from a perfusor syringe, from which the plunger had been removed. The open end of the syringe was securely placed over the nose of the rat. The depth of anesthesia could be modified by changing isoflurane concentration. The following schedule was used: Carbofen ${ }^{\circledR} 4 \mathrm{mg} / \mathrm{ml}$ s.c. preoperatively on the day the operation was performed followed by a single daily dose on the 2 nd and 3rd postoperative days. Postoperative analgesia was done with novaminsulfon $\left(\right.$ Metamizol $\left.^{\circledR}\right) 50 \mathrm{mg} / \mathrm{kg}$ i.m., and every $6 \mathrm{~h}$ the animals received 2 drops p.o. until the $3 \mathrm{rd}$ postoperative day.

\section{Donor Surgical Technique}

Preparation of the Pancreas

After shaving the abdominal wall a median laparotomy was performed. The laparotomy site was secured with four abdominal retractors and the xiphoid was fixed cranially. First, the tail of the pancreas was prepared and the spleen was mobilized. After removing the pancreas from the intestinal adhesions, the duodenum was ligated below the head of the pancreas and taken out (fig. 1). After surgical exposition of the celiac axis and the superior mesenteric artery, the abdominal aorta was prepared, and the left gastric artery and the hepatica artery were identified and ligated. At this stage, arterial perfusion of the pancreas and the duodenal segment was ensured via superior mesenteric artery, celiac axis, gastroduodenal and splenic arteries. Finally, the vena porta was dissected following ligation of the splenic vein. Venous outflow was then maintained by inferior mesenteric vein and portal vein.

Preparation of the Kidney

Dissection of the right kidney started from the margo lateralis to the central hilum. After removing the fatty tissue surrounding 
Fig. 2. The organs were then removed and kept in cold preservation solution at $4^{\circ} \mathrm{C}$ for $1 \mathrm{~h}$ (pancreas preservation).

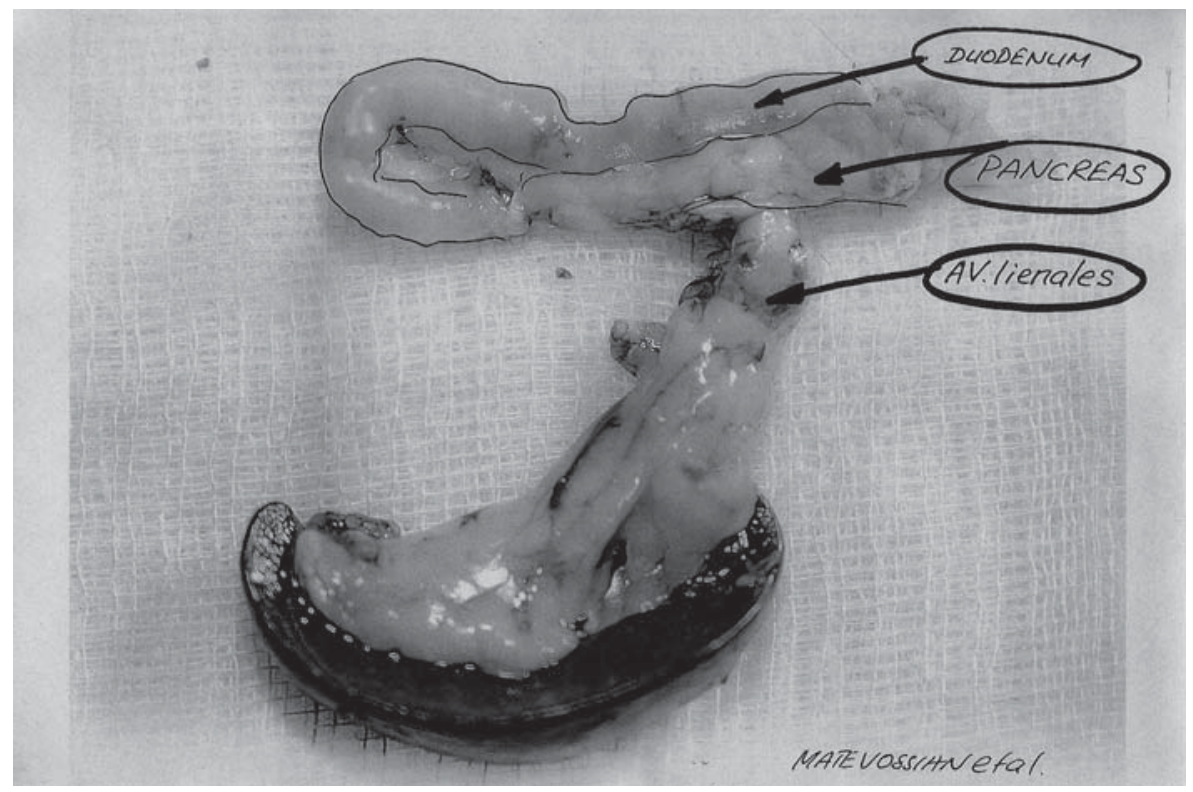

renal arteries, the veins and the ureter were identified and ligated. The blood vessels were cut leaving a maximum stump length and the ureter was harvested with a patch of bladder tissue. Continuous urine outflow from the ureter was a reliable sign to monitor kidney function.

Perfusion and Explantation of Pancreas and Kidney

To begin the perfusion of the donor operation, a microvessel clip (Mehdorn-Biemer, Aesculap AG\&Co. KG, Tuttlingen, Germany) was placed on the aorta above the celiac axis. A 24-gauge perfusion catheter (Venflon 2, ABOC Health Care Company, Helsingborg, Sweden) was inserted into the aorta. The perfusion was performed with cold Ringer's lactate fluid at $4^{\circ} \mathrm{C}$ with 50 IU heparin. Perfusion pressure was $100 \mathrm{~mm} \mathrm{Hg}$, which is equal to the physiologic aortic pressure of the rat. $40 \mathrm{ml}$ of the perfusion fluid was administered, corresponding to the 6 -fold organ weight for equilibration. The suprahepatic vena cava was then incised to enable blood and perfusion outflow. The organs were then removed and kept for $1 \mathrm{~h}$ in cold preservation solution at $4^{\circ} \mathrm{C}$ (fig. 2).

\section{Recipient Surgical Technique}

Organ Transplantation (Pancreas)

The transplantation procedure began identically to the donor operation with the aorta clamped distal to the celiac axis using microclips. The recipient's pancreas was completely isolated by interrupting arterial and venous blood flow by ligature and electrocoagulation after laparotomy and abdominal exposition (ligatures: Aa. pancreaticoduodenalis superior et inferior; electrocoagulation: the rest of the vessels). Anastomosis of the abdominal aorta (recipient) with the aortic segment of the donor was performed using a running suture in side-to-end technique in $8 \pm$ 1.5 min (mean $\pm \mathrm{SD}$ ) (fig. 3 ). In the same way, the venous anastomosis of a vena cava segment to the vena cava (recipient) was completed in $6 \pm 1 \mathrm{~min}$. After declamping of the aorta and vena cava, reperfusion started. The warm ischemic time was $17 \pm 2.5$ min. The position of the transplanted pancreas was in the right upper abdomen and the duodenal segment was connected sideto-side with the jejunum of the recipient through a running suture with 7/0 Prolene (fig. 4). Finally, the anastomosis was covered with the omentum.

\section{Kidney}

The recipient's kidneys were isolated by ligature of the renal arteries and veins. To prevent urinary reflux, the recipient's right and left ureters were ligated. After dissection of the aorta and the inferior vena cava segment, both arteries and veins of the kidneys were ligated and the recipient showed acute renal dysfunction. Now the aortic and venous patches on the allograft kidney were anastomosed to the aorta and the vena cava. The time for anastomosis was $5 \mathrm{~min}$ on the arterial side and $6 \mathrm{~min}$ for the vein. The median warm ischemic time was $14 \pm 2.0 \mathrm{~min}$. After declamping, the kidney showed complete reperfusion with a homogeneous pink color. Ten minutes later, all recipients showed urine production via the ureter. Finally, the ureter was inserted into the bladder with a single-stitch suture (9/0 Ethilon, not resorbable, monofil, Ethicon, Germany).

\section{Postoperative Care}

After the transplantation, the recipients were put into single cages with infrared radiation, and received water and food. For 12 days postoperatively, the recipients were observed and the body weight and the health status were documented. Daily laboratory analyses (creatinine, urea, lipase, amylase and blood glucose) were performed. To ensure regular hemodilution, the recipients received daily $2 \mathrm{ml}$ physiological $\mathrm{NaCl} 0.9 \%$. On day 5 and day 8 , biopsies from the transplanted organs were obtained by minilaparotomy. On day 12, recipients were sacrificed after final venous blood analysis and registration of the body weight. A blood plasma sample was shock-frozen and stored in liquid nitrogen $\left(-80^{\circ} \mathrm{C}\right)$. Transplanted organs were removed for histopathological examination and fixed in $3.7 \%$ buffered formalin solution (Merck, Darmstadt, Germany). 
Fig. 3. Anastomosis between abdominal aorta (recipient) and the aortic segment of the donor was performed with a running suture using side-to-end technique.
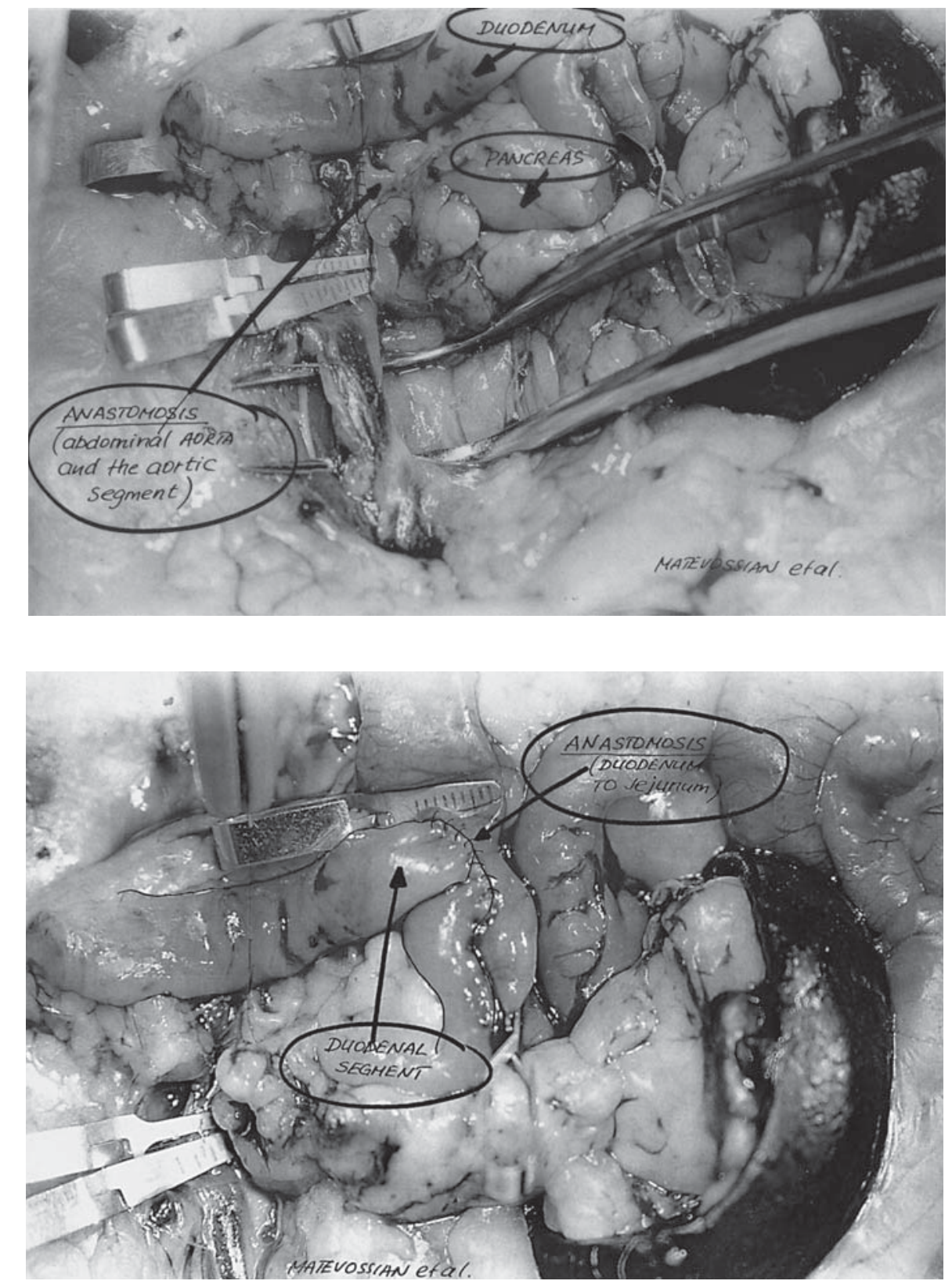

Fig. 4. The position of the transplanted pancreas was in the right upper abdomen and the duodenal segment was connected side-to-side with the jejunum of the recipient through a running suture. Finally the anastomosis was covered with the omentum.

\section{Results}

In all transplanted pancreas organs on postoperative day 5 , cell infiltration between the lobes with macrophages and some granulocytes was found histopathologically. On day 8, regular pancreas tissue was diagnosed without inflammatory infiltrations (fig. 5). In the duodenal segment in two cases a small villous atrophy was seen with no other pathological signs (fig. 6). On day 12, four transplanted pancreas grafts showed interlobular cell in- filtration, consisting of lymphocytes and macrophages, spreading to the exocrine pancreas tissue. At that time point, in all organs, proliferation of fibroblasts and isolated macrophages on the endothelium, but without endothelialitis, were found. In 1 recipient, irreversible graft loss occurred by thrombosis of the portal vein (outflow of the transplanted pancreas). The recipient's own pancreas and kidneys showed total necrosis on day 5 after transplantation. All kidney grafts showed excellent perfusion at day 5. No complications such as bleeding, 


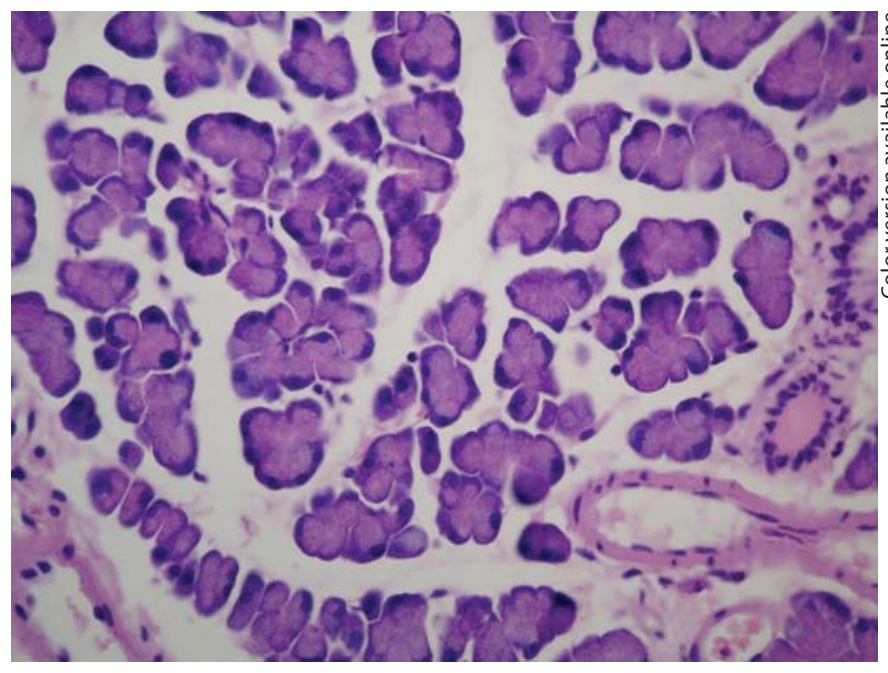

Fig. 5. On day 8 , regular pancreatic tissue was diagnosed without inflammatory infiltrations. Hematoxylin and eosin staining. Magnification $\times 400$.

thrombosis or kinking were seen in the other 11 cases (fig. 7). Serum creatinine dropped to $1.7 \pm 0.3 \mathrm{mg} / \mathrm{dl}$ on day 3 and reached normal values $(0.9 \pm 0.1 \mathrm{mg} / \mathrm{dl})$ on day 5 (table 1). A similar course was found for urea.

In all recipients blood glucose was normal on day 5 after transplantation $(5.7 \mathrm{mmol} \pm 0.5)$; however, on postoperative day 9 , hyperglycemia caused by thrombosis of the portal vein from the pancreas was diagnosed (8.0 $\mathrm{mmol}$ ) in 1 recipient. During the first 3 days, all animals showed weight loss of $25 \%$, which they subsequently regained 12 days later.

\section{Discussion}

The combination of pancreas and kidney transplantation in selected patients with type I diabetes, and hemodialysis is the therapy of choice [5-7]. However, despite its clinical success, combined pancreas/kidney transplantation is a major operation, and its results were formerly associated with high morbidity [6]. By now, substantial improvements in transplant medicine have led to more than 21,000 transplanted pancreas organs worldwide. Technical and immunological features of the combined PKTx and resulting complications underline the necessity of experimental research, especially transplant models identical with clinical aspects of transplantation.

The transplantation model presented here achieves perfect simulation of the clinical process, and is easily

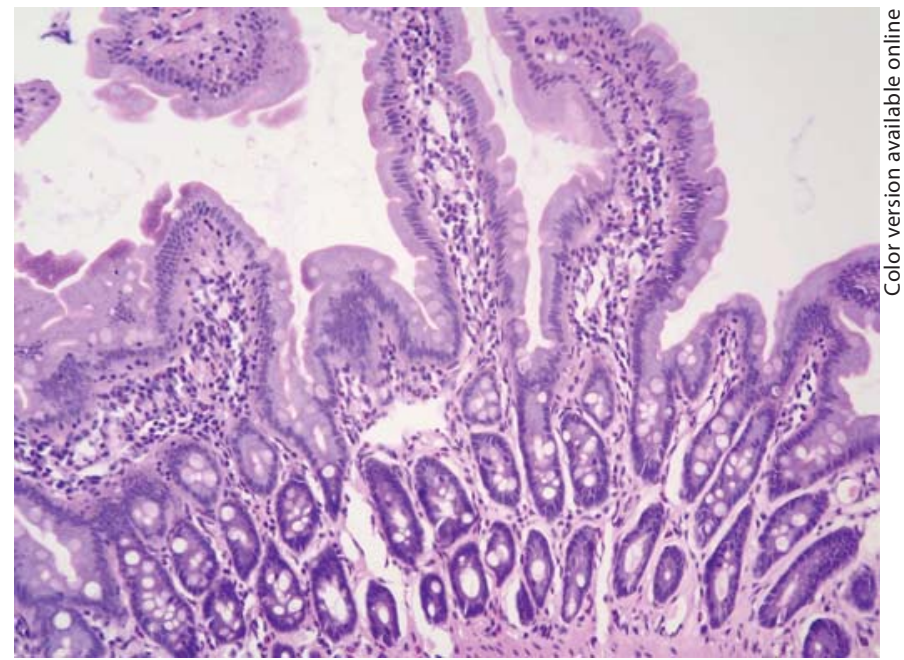

Fig. 6. In the duodenal segment of 2 cases, a small villous atrophy was seen with otherwise no pathological signs. Hematoxylin and eosin staining. Magnification $\times 200$.

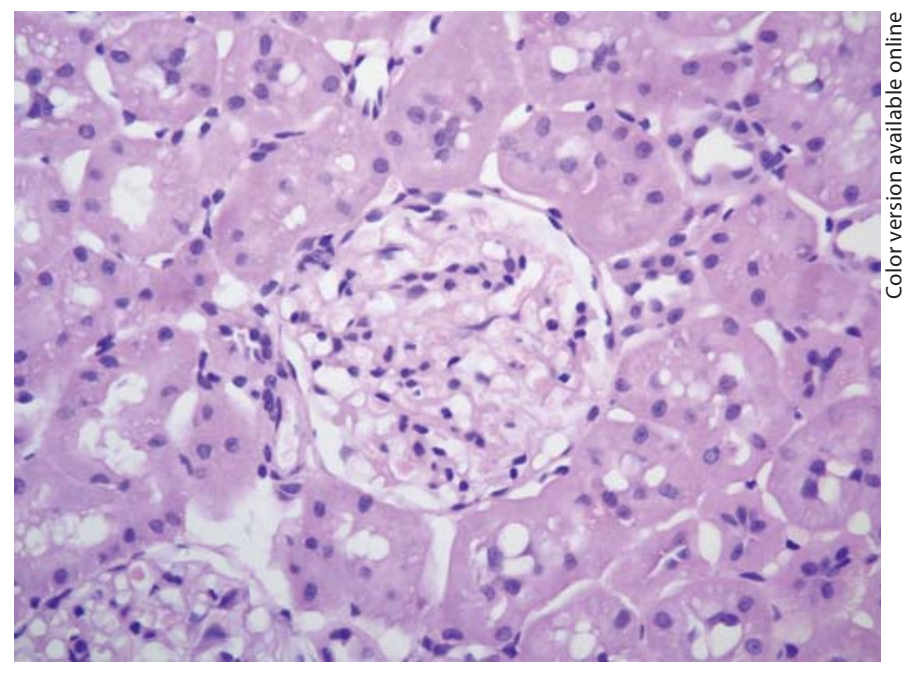

Fig. 7. All kidney grafts showed excellent perfusion on day 5 . Hematoxylin and eosin staining. Magnification $\times 400$.

Table 1. Plasma values after transplantation on days 5,8 and 12 ( \pm standard deviation; $\mathrm{n}=12$ )

\begin{tabular}{llll}
\hline $\begin{array}{l}\text { Day after PKTx } \\
(\mathrm{n}=12)\end{array}$ & $\begin{array}{l}\text { Creatinine } \\
\mathrm{mg} / \mathrm{dl}\end{array}$ & $\begin{array}{l}\text { Blood glucose } \\
\mathrm{mmol} / \mathrm{l}\end{array}$ & $\begin{array}{l}\text { Lipase } \\
\mathrm{U} / \mathrm{l}\end{array}$ \\
\hline Day 5 & $0.97 \pm 0.1$ & $5.7 \pm 0.5$ & $201.0 \pm 13.5$ \\
Day 8 & $0.85 \pm 0.09$ & $5.5 \pm 0.7$ & $143.7 \pm 12.2$ \\
Day 12 & $0.8 \pm 0.1$ & $4.9 \pm 0.6$ & $126.4 \pm 14.0$ \\
\hline
\end{tabular}


reproducible with low rates of mortality and complication, resulting in high graft survival. Therefore, this model has high clinical relevance to technical and immunological issues of transplantation medicine. The technique developed and described here is reliable and presents the first report of this method of combined PKTx. The single pancreas transplantation was first regarded as experimental therapy for patients with type I diabetes for two decades since its first description in 1967, because graft survival compared to liver and kidney transplanted patients were clearly worse [6]. Due to progress in transplantation medicine, currently more than 21,000 single pancreas transplantations with patient and graft survival in 95 and $87.5 \%$ combined with kidney transplantation were registered in the International Pancreas Transplant Registry [5-7]. Since the first successful kidney transplantation in Boston in 1954 between identical twins, more than half a million patients in 1,400 specialized centers underwent transplantation $[8,14]$. The special quality of technique and immunology of the combined PKTx requires experimental basic research. In particular, standardized and reproducible models which reflect technical aspects are needed. Transplanted pancreas organs with intestinal drainage of exocrine secretion in the syngeneic animal transplantation model have an advantage over models without drainage of exocrine secretion. All morphologic alterations due to this technique were considered to be a consequence of graft rejection, and in iso-transplanted organs this will be interpreted as technical failure $[2-4,15,16]$. Formerly, the drainage of secretion in pancreas transplantation was ensured via the duodenum of the donor, in which the oral side was closed and the aboral side was connected end-to-side with the first jejunal loop of the recipient [4]. For morphologic examination of rejection signs, a model with intestinal drainage is thought to be more favorable according to established clinical procedures. This is mainly because lymphatic cell infiltration and remodelling processes as observed in syngeneic transplants would not be detected in pancreaticoduodenal transplants [11]. The transplant model presented here resembles side-to-side duodenojejunostomy that is tailored to the clinical procedure.

Furthermore, the model of combined PKTx allows the comparison of different transplantation components of the pancreas, kidney and duodenum with respect to rejection characteristics. Also concerning transplantation order, time factor and exocrine pancreas drainage the model is comparable to the daily clinical course. Vascular anastomosis with the aorta and inferior vena cava of both transplanted organs as described has mainly been per- formed in children, but there is no disadvantage compared to the technique in adults by using the external iliac artery. Arterial inflow is provided through the celiac axis and venous outflow through the inferior vena cava. Thus complications of the portal anastomosis, the number of anastomoses and operation time will be markedly reduced [3, 4, 17-20]. In one recipient venous thrombosis of the vena portae occurred, and the graft was lost 10 days after transplantation. Typical complications, such as postoperative bleeding, kinking of the vessels, pancreatitis or anastomotic leak, were not observed.

Research in the field of transplantation immunology in small animal models is adapted to clinical praxis. Organ transplantation in rats is a well-established procedure because the genetic characteristics have been investigated, and body weight allows the transplantation of vascularized organs using microsurgical technique [4]. On the basis of clinical transplantation microsurgical aspects of combined PKTx were presented in detail using an experimental model and illustrated with original pictures and drawings.

\section{Acknowledgments}

We sincerely thank Dr. Thomas Brill (MVD, MD), Dr. Anne Preissel (MVD, MD) (Department of Preclinical Research, Technische Universität München) for excellent anesthesiological and technical assistance. The authors also acknowledge the assistance of Ms. Jennifer Altomonte (MD, University of New York) for critical reading of the manuscript.

References

1 Thide A, Brieler HS, Delitz R, et al: Zur experimentellen Mikrochirurgie an Ratten. Zentralbl Chir 1978;103:1397.

2 Benetti L, Bassi C, Zamboni G, et al: Pancreaticoduodenal graft in the rat: an original micro-surgical technique. Eur Surg Res 1989;21:162.

3 Lee S, Scott M, Macedo AR: Pancreaticoduodenal transplantation in the rat: a technique update. Transplantation 1986;41:327.

4 Lee S, Tung KS, Koopmans H, et al: Pancreaticoduodenal transplantation in the rat. Transplantation 1972;13:421.

5 Gruessner AC, Sutherland DE: Pancreas transplant outcomes for United States (US) and non-US cases as reported to the United Network for Organ Sharing (UNOS) and the International Pancreas Transplant Registry (IPTR) as of June 2004. Clin Transpl 2005; 19:433.

6 International Pancreas Transplant Registry. http//:www.iptr.umn.edu. 
7 Kelly WD, Lillehei RC, Merkel FK, et al: Allotransplantation of the pancreas and duodenum along with the kidney in diabetic nephropathy. Surgery 1967;61:827.

8 US Organ Procurement and Transplantation Network and the Scientific Registry of Transplant Recipients. http//:www.optn.org.

9 Agnes S, Castagneto M: Transplantation of the isolated pancreas in rats: technical aspects. Minerva Chir 1980;31:683.

10 Di Cataldo A, Puleo S, Li Destri G, et al: Pancreas transplantation in the rat using Lee's technique: a reliable model in experimental microsurgery. J Invest Surg 1989;2:159.

11 Königsrainer A, Habringer C, Krausler R, et al: A technique of pancreas transplantation in the rat securing pancreatic juice for monitoring. Transpl Int 1990;3:181.
12 Knoop M, Steffen R, Neuhaus P: A technique for hepatopancreaticoduodenal cluster transplantation in the rat. Microsurgery 1991;12:385

13 Mood ZA, Mehrabi A, Schmied BM, et al: Review of various techniques of pancreas transplantation in rat model. J Surg Res 2008;145:205-213.

14 Murray JE, Merril JP, Harrison JH: Renal homotransplantation in identical twins. Surg Forum 1955;6:432.

15 Marni A, Ferrero ME: Simultaneous transplantation of kidney and duct-ligated segmental pancreas in the rat: en bloc technique using two vascular anastomoses. Transplantation 1984;37:544.

16 Yoon JN, Jung SI, Cho C, et al: Effects of simultaneous kidney-pancreaticoduodenal transplantation on diabetes-induced renal insufficiency in rats. Microsurgery 2001;21: 173.
17 Lugagne PM, Reach G, Houry S, et al: Portal versus peripheral venous drainage in segmental pancreatic transplantation in diabetic rats. Transplantation 1989;47:420.

18 Timmermann W, Schlang T, Stoffregen C, et al: Development and perspectives of experimental pancreas transplantation in the rat. Microsurgery 1990;11:133.

19 Ohtsuka S, Yokoyama I, Hayashi S, et al: Experimental rat pancreas transplant: surgical technique and immunological considerations. Surg Today 1994;24:247.

20 Sanchez de Badajoz E, Vara Thorbeck C: Pancreatic transplantation in the rat: an experimental model. Z Exp Chir Transplant Kunstliche Organe 1990;23:26. 